Ophthalmologica

\title{
Astrocytoma of the Ciliary Body
}

\author{
Haifeng Mei ${ }^{\mathrm{a}}$ Yiqiao Xing ${ }^{\mathrm{a}}$ Anhuai Yang $^{\mathrm{a}}$ Jing Wang ${ }^{\mathrm{a}} \mathrm{YaXu}^{\mathrm{a}}$ \\ Zesheng Wang $^{\mathrm{b}}$ Arnd Heiligenhaus $^{\mathrm{c}}$
}

Departments of a Ophthalmology and ${ }^{b}$ Pathology, Renmin Hospital of Wuhan University, Wuhan, PR China;

'Department of Ophthalmology at St. Franziskus Hospital, University of Duisburg-Essen, Münster, Germany

\section{Key Words}

Astrocytoma $\cdot$ Tumor $\cdot$ Ciliary body

\begin{abstract}
Purpose: To report the occurrence of an astrocytoma of the ciliary body. Methods: The patient was evaluated by ophthalmoscopic examination (including color ultrasonography and ultrasound biomicroscopy) as well as operating therapy. Histopathology and immunocytochemical analysis were performed. Results: A 29-year-old woman was found to have a left ciliary body tumor. Color ultrasonography revealed a $12 \times 10 \mathrm{~mm}$ size, a low-level echo boundary, and a bright echogenic band. Ultrasound biomicroscopy showed that the lesion was located in the superior temporal quadrant of the ciliary body and extended backward for $6 \mathrm{~mm}$ from the limbus. The tumor was carefully dissected free and completely removed via episcleral incision. Histopathology revealed a fibrillary astrocytoma. Staining for glial fibrillary acidic protein yielded positive results and neuron-specific enolase yielded negative results. Conclusions: We describe an astrocytoma of the ciliary body in a patient, which is an extremely rare ocular tumor. Histopathology and immunocytochemical analysis were required to establish the diagnosis.

Copyright $\odot 2008$ S. Karger AG, Basel
\end{abstract}

\section{Introduction}

Gliomatous tumors arising from the retina and optic nerve are rare, especially when located in the ciliary body. To our knowledge, there are only two case reports of astrocytomas of the ciliary body. In this study, we report a patient with an astrocytoma of the ciliary body in whom histopathology and immunocytochemical analysis established the diagnosis.

\section{Case Report}

A 29-year-old woman complained about loss of visual acuity of her left eye over half a year. On ophthalmic examination, her visual acuity was 20/20 OD and 20/40 OS. The intraocular pressures (IOP) were $14 \mathrm{~mm} \mathrm{Hg} \mathrm{OD} \mathrm{and} 19 \mathrm{~mm} \mathrm{Hg}$ OS. A dilated, indirect ophthalmoscopic examination revealed a highly prominent brown, vascularized, solid homogenous lesion in the ciliary body at the 1:30 to 4:30 o'clock meridian. Examination of the optic disk and retina indicated normal results. Transillumination of the globe revealed a shadow at the ciliary body.

Color ultrasonography showed a low-level echo in the temporal quadrant of the left eye located at the posterior border of the ciliary body (size $12 \times 10 \mathrm{~mm}$ ). The low-level echo boundary was distinct with a bright echogenic band and its inferior border was uniform (fig. 1).

Ultrasound biomicroscopy showed that the lesion was located in the superior temporal quadrant of the ciliary body and extended backward for $6 \mathrm{~mm}$ from the limbus (fig. 2).

After a complete medical examination, the tumor was carefully dissected free and completely removed via episcleral incision (fig. 3). Histopathology revealed a fibrillary astrocytoma.

\section{KARGER \\ Fax +4161306 1234 E-Mail karger@karger.ch} www.karger.com
E-Mailmhf021471@yahoo.com 

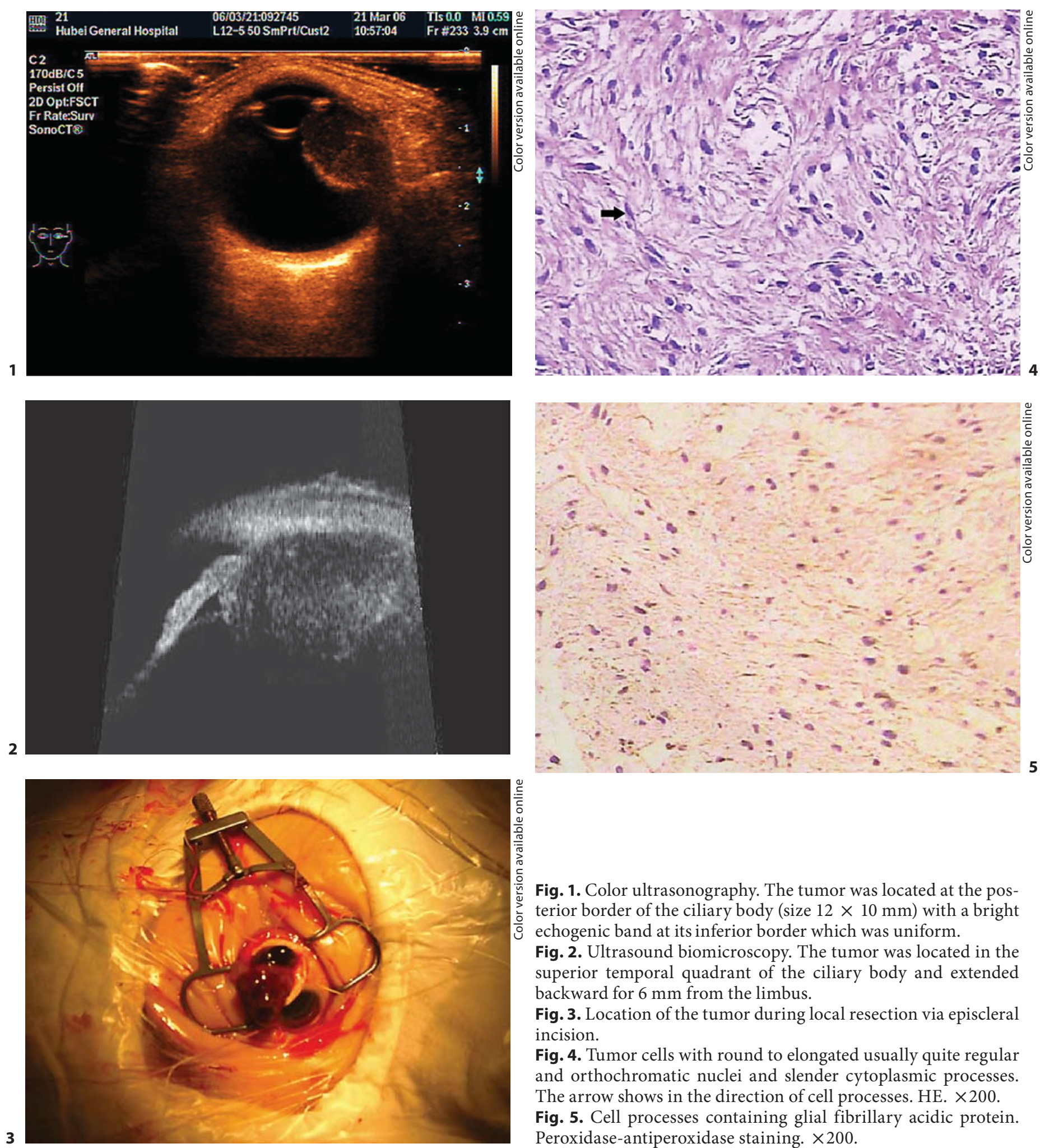

Fig. 1. Color ultrasonography. The tumor was located at the posterior border of the ciliary body (size $12 \times 10 \mathrm{~mm}$ ) with a bright echogenic band at its inferior border which was uniform.

Fig. 2. Ultrasound biomicroscopy. The tumor was located in the superior temporal quadrant of the ciliary body and extended backward for $6 \mathrm{~mm}$ from the limbus.

Fig. 3. Location of the tumor during local resection via episcleral incision.

Fig. 4. Tumor cells with round to elongated usually quite regular and orthochromatic nuclei and slender cytoplasmic processes. The arrow shows in the direction of cell processes. HE. $\times 200$.

Fig. 5. Cell processes containing glial fibrillary acidic protein. Peroxidase-antiperoxidase staining. $\times 200$. 
No complications were noted during the operative and postoperative course. One month after surgery, the visual acuity of her left eye was stable at 20/20. IOP was $13 \mathrm{~mm} \mathrm{Hg}$. Results of the vitreous and peripheral retinal examination were normal.

The tumor was a well-differentiated diffusely infiltrating neoplasm of fibrillary astrocytes. The nuclei were round to oval and fibrillary cell processes were thin and elongated. Slight and definite variations in nuclear size and shape were seen in tumor cells (fig. 4).

Immunocytochemical staining for glial fibrillary acidic protein on formalin-fixed, paraffin-embedded tissue revealed positive staining (fig. 5). Neuron-specific enolase staining yielded negative results.

\section{Discussion}

Gliomatous tumors arising from the retina and optic nerve are very rare, especially when located in the ciliary body. To our knowledge, there are only two case reports of astrocytomas of the ciliary body [1].
Astrocytoma of the ciliary body may be a pathological change of choristoma in the anterior lip of the optic cup. However, the mechanism of it is still unclear. Someone speculated it would present from birth but later develop vascular incompetence with accumulation of pools and lakes of eosinophilic material [2]. Another viewpoint held that it must arise from normal ciliary epithelium as a metaplastic process [3]. Farber et al. [4] considered that the tumor represented a congenital lesion arising from the nonpigmented epithelium and belonged in the spectrum of glioneuromas and medulloepitheliomas.

Astrocytoma of the ciliary body is a benign tumor which represents the fist stage of astrocytoma. There is no malignant report of it. Local resection of the lesion via episcleral incision is a good way to successfully preserve a viable globe. Complete resection of the lesion results in the preservation of good visual acuity and a normal IOP.
References
3 Kuhlenbeck H, Haymaker W: Neuroectodermal tumors containing neoplastic neuronal elements: ganglioneuroma, spongioneuroblastoma, and glioneuroma. Mil Med 1946;99:273-292. Acta Ophthalmol (Copenh) 1985;63:28-30.

-2 Spencer WH, Jesberg DO: Glioneuroma (choristomatous malformation of the optic cup margin): a report of two cases. Arch Ophthalmol 1973;89:387-391.
4 Farber MG, Smith ME, Gans LA: Astrocytoma of the ciliary body. Arch Ophthalmol 1987; 105:536-537. 